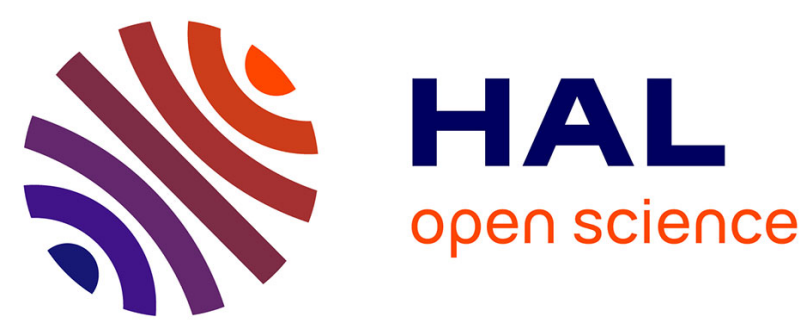

\title{
Determining the size of RVE for nonlinear random composites in an incremental computational homogenization framework
}

\author{
T.H. Hoang, M. Guerich, Julien Yvonnet
}

\section{- To cite this version:}

T.H. Hoang, M. Guerich, Julien Yvonnet. Determining the size of RVE for nonlinear random composites in an incremental computational homogenization framework. Journal of Engineering Mechanics ASCE, 2016, 142 (5), pp.04016018. 10.1061/(ASCE)EM.1943-7889.0001057 . hal-01228966

\section{HAL Id: hal-01228966 https://hal.science/hal-01228966}

Submitted on 15 Nov 2015

HAL is a multi-disciplinary open access archive for the deposit and dissemination of scientific research documents, whether they are published or not. The documents may come from teaching and research institutions in France or abroad, or from public or private research centers.
L'archive ouverte pluridisciplinaire HAL, est destinée au dépôt et à la diffusion de documents scientifiques de niveau recherche, publiés ou non, émanant des établissements d'enseignement et de recherche français ou étrangers, des laboratoires publics ou privés. 


\title{
Determining the size of RVE for nonlinear random composites in an incremental computational homogenization framework
}

\author{
T.H. Hoang a,b, M. Guerich ${ }^{\mathrm{a}, *}$, J. Yvonnet ${ }^{\mathrm{b}}$, \\ ${ }^{a}$ Département de Mécanique Numérique et Modélisation, Technology Lab., Ecole \\ Supérieure d'Ingénieurs Léonard de Vinci (ESILV), 92916 Paris la Défense \\ Cedex, France \\ ${ }^{\mathrm{b}}$ Université Paris-Est, Laboratoire Modélisation et Simulation Multi Échelle \\ MSME UMR 8208 CNRS, 5 bd Descartes, F-77454 Marne-la-Vallée, France.
}

\begin{abstract}
In this paper, we address the issue of determining the size of a representative volume element (RVE) in the case of nonlinear random composites with either elastoplastic or elasto-viscoplastic phases. In such a case, the general form of the effective constitutive behavior is not known in advance and the response must be evaluated either by direct numerical computations on the RVE, either by an appropriate approximation scheme. Previous methodologies for determining the size of RVE usually rely on analyzing the convergence of the RVE response computed numerically with respect to its size. In the present work, we analyze the convergence of parameters related to an incremental homogenization scheme, with respect to (i) the size of the RVE and (ii) to statistical convergence related to microstructure realizations. For that purpose, we combine an incremental homogenization method with a statistical convergence analysis of parameters related to the matrix phase only. The advantage is that the range of parameters to be identified is much narrower than for a general empirical constitutive law. Once identified and the convergence analysis performed with respect to both size of RVE and statistical realizations, the macroscopic constitutive law can be readily used for structure calculations. We illustrate the methodology by analyzing two-dimensional microstructures with randomly distributed cylindrical elastic rigid fibers, embedded in a elastoplastic or elasto-viscoplastic matrix. For these materials, the existence of an RVE is demonstrated for sizes of RVE corresponding to $17-18$ and $14-15$ times the diameter of the inclusions, respectively.
\end{abstract}

Key words: Computational homogenization, Size of RVE, nonlinear composites, Incremental homogenization. 


\section{Introduction}

A fundamental concept in homogenization of composites is that of Representative Volume Element (RVE), determining the domain associated with the microstructure to evaluate the effective behavior of the heterogeneous materials. If this notion is nowadays well defined for random linear composites, many issues remain when considering nonlinear heterogeneous materials. A review of the different definitions of an RVE can be found in [9] for linear and nonlinear materials. Several statistical processes have been proposed, including, among others, the procedures of Kanit et al. [15], Gitman et al. [9], or Pelissou et al. $[20]$.

A first class of methods for studying the size of RVE includes approaches based on analytical homogenization schemes, mainly restricted to linear cases. These techniques (see e.g. $[17,21,33]$ ), have been used to consider spherical or spheroidal-shaped inclusions and have been useful in some situations to determine the size of the RVE with respect to the inclusions characteristic size.

A second class of approaches, based on numerical methods such as the finite element method (FEM) (see, e.g., among many others, [2,10,22,25,31]), uses computations on a unit cell and allows determining the size of the RVE via statistical analyses relying on numerical computations. These techniques have been mainly applied in the linear case, and a few recent studies involve nonlinear heterogeneous materials. For linear composites, determining the size of the RVE can be performed by analyzing the statistical convergence of effective material parameters with respect to the size of the unit cell. In [15], Kanit et al. studied the linear thermal and elastic properties of random 3D polycristalline microstructures. In [23], Ostoja-Starzewski et al. investigated random polycristal microstructures made-up of cubic single crystals. Other examples in elasticity can be found in $[7,12,18,24,34]$. Applications to cortical bone, molecular dynamics models of polymer or porous media have been studied in $[5,11,32]$. In [28], new criteria to determine the size of RVE with random elastic matrix have been proposed as well as estimates for RVE sizes. In [30], a stochastic homogenization theory has been introduced for random anisotropic elastic composites which cannot be described in terms of their constituents and for which the standard methods cannot be applied, like cortical bones or biological membranes. In [26], a method using the concept of periodization of random media was used to estimate the effective properties of random composites using small volumes.

For nonlinear compos, most of the proposed methodologies are based on an-

\footnotetext{
* Correspondance to M. Guerich

Email address: mohamed.guerich@devinci.fr (M. Guerich ).
} 
alyzing the convergence of the effective response (e.g. the effective stress), computed numerically at one point of the loading curve, with respect to the size $[3,13,27]$. More recent studies analyze the convergence of identified parameters related to an empirical macroscopic model with respect to the unit cell size $[16,20]$.

In the present paper, a new methodology to estimate the size of the RVE for nonlinear composites is proposed. The technique combines statistical analysis based on the convergence of identified macroscopic parameters and homogenization. An incremental homogenization technique [4] is employed, which provides the effective constitutive behavior of the material. Then, the convergence of parameters associated with the homogenization process is analyzed with respect to the RVE size through an appropriate metric. The advantages of the present framework are twofold: the size of the RVE for nonlinear materials like elastoplastic or elasto-viscoplastic ones can be estimated; (b) the converged parameters can be used to efficiently compute the effective constitutive law through the incremental homogenization scheme for structure calculations.

The paper is organized as follows. In section 2 , the incremental homogenization method for nonlinear materials is reviewed. In section 3 , the proposed statistical process is presented. In section 4 , the technique is applied to the analysis of random nonlinear fiber-composites whose matrix has either a elastoplastic or an elasto-viscoplastic behavior.

\section{Review of the incremental homogenization method}

Incremental homogenization schemes are extensions of the formulation proposed by Hill [14] in which macroscopic stress and strains are related by a constitutive law in the form:

$$
\dot{\overline{\boldsymbol{\sigma}}}(t)=\overline{\mathbb{C}}(t): \dot{\bar{\varepsilon}}(t)
$$

where $\dot{\overline{\boldsymbol{\sigma}}}$ and $\dot{\overline{\boldsymbol{\varepsilon}}}(t)$ are rates of macroscopic stress and strain, respectively and $\overline{\mathbb{C}}(t)$ is the tangent operator depending on both the actual strain state and on the history of loading. For the linearized problem, it is possible to apply the superposition principle and to compute the effective tangent operator $\overline{\mathbb{C}}(t)$ at each iteration, by the knowledge of the nonlinear constitutive law in each phase and of the strain at the former iteration. Let $\Omega$ be a domain in $\mathbb{R}^{d}$ associated to the RVE, $d$ being the space dimension. The boundary of $\Omega$ is denoted by $\partial \Omega$. We assume that $\Omega$ contains two phases, the matrix, associated to a domain $\Omega_{0}$ and the inclusion, defined in a domain $\Omega_{1}$. The interfaces between the phases are assumed to be perfectly bounded. 
For a given analytical homogenization scheme (e.g. self-consistent, Mori-Tanaka, see e.g. [6]), given the strain increment $\Delta \bar{\varepsilon}$ prescribed on the RVE at time $t^{n}$, it is possible to evaluate the tangent modulus associated to the nonlinear constitutive laws in each phase (see e.g. $[4,1,29]$ ), which are used to compute the effective tangent modulus at time $t^{n+1}$. For example, in the scheme proposed by Doghri et al. [4], the problem is to determine at time $t^{n+1}$ the average strain in the inclusions, which is evaluated iteratively. Let $\langle\Delta \varepsilon\rangle_{\Omega}=\Delta \bar{\varepsilon}$ be the strain increment, denoted at time $t^{n}$ by $\left\langle\Delta \varepsilon_{n}\right\rangle_{\Omega}=\Delta \bar{\varepsilon}_{n}$. The symbol $\langle.\rangle_{\omega}=\frac{1}{|\omega|} \int_{\omega}(). d \Omega$ is the spatial averaging over a domain $\omega$. The matrix and inclusions are associated to the indices 0 and 1, respectively. The average strain in the matrix and in inclusions are denoted by $\langle\Delta \varepsilon\rangle_{\Omega_{0}}$ and $\langle\Delta \varepsilon\rangle_{\Omega_{1}}$, respectively. For a new increment of $\left\langle\Delta \varepsilon_{n}\right\rangle_{\Omega_{1}}$, the average strain in the matrix $\langle\Delta \varepsilon\rangle_{\Omega_{0}}$ can be computed by

$$
\Delta \bar{\varepsilon}=\langle\Delta \varepsilon\rangle_{\Omega_{0}}\left(1-f_{1}\right)+f_{1}\langle\Delta \varepsilon\rangle_{\Omega_{1}}
$$

where $f_{1}$ denotes the volume fraction of inclusion. Using the expression of the tangent modulus computed in each phase, the Eshelby tensor $\mathbb{E}$ can be evaluated (see e.g. [6]). We can then compute the concentration tensor $\mathbb{B}^{\epsilon}$ to relate the average strain in each phase to the macroscopic strain by

$$
\begin{aligned}
& \langle\Delta \varepsilon\rangle_{\Omega_{0}}=\left[f_{1} \mathbb{B}^{\epsilon}+\left(1-f_{1}\right) \mathbb{I}\right]^{-1}: \bar{\varepsilon} \\
& \langle\Delta \varepsilon\rangle_{\Omega_{1}}=\mathbb{B}^{\epsilon}:\left[f_{1} \mathbb{B}^{\epsilon}+\left(1-f_{1}\right) \mathbb{I}\right]^{-1}: \bar{\varepsilon}
\end{aligned}
$$

where $\mathbb{B}^{\epsilon}$ is expressed by

$$
\mathbb{B}^{\epsilon}=\left\{\mathbb{I}+\mathbb{E}:\left[\mathbb{C}_{0}^{-1}: \mathbb{C}_{1}-\mathbb{I}\right]\right\}^{-1},
$$

where $\mathbb{C}_{0}$ and $\mathbb{C}_{1}$ are the tangent moduli associated to the nonlinear constitutive laws in each phase. Eventually, the effective tangent modulus is expressed by

$$
\overline{\mathbb{C}}=\left[f_{1} \mathbb{C}_{1}: \mathbb{B}^{\epsilon}+\left(1-f_{1}\right) \mathbb{C}_{0}\right]:\left[f_{1} \mathbb{B}^{\epsilon}+\left(1-f_{1}\right) \mathbb{I}\right]^{-1}
$$

For a Mori-Tanaka scheme, the algorithm proposed by Doghri et al. in [4], is described as follows. Let $\left[t_{n}, t_{n+1}\right]$ a time interval. Given $\overline{\boldsymbol{\varepsilon}}_{n}, \Delta \overline{\boldsymbol{\varepsilon}}_{n}$ and internal variables in phases at time $t_{n}$, the problem consists in determining the overall stress $\overline{\boldsymbol{\sigma}}_{n+1}$ and the tangent modulus $\overline{\mathbb{C}}_{n+\alpha}$, where $n+\alpha$ denotes the time $t^{n+\alpha}=t^{n}+\alpha \Delta t$. The different steps of the algorithm are summarized as follows:

- Initialization $\langle\bar{\varepsilon}\rangle_{\Omega_{1}}=\Delta \bar{\varepsilon}$.

- WHILE $\|\mathbf{R}\|>T O L$, where $T O L$ is a numerical tolerance:

(1) Iteration $k$ (upper index $k$ is omitted for the sake of simplicity)

(2) Given $\left\langle\varepsilon_{n}\right\rangle_{\Omega_{1}}$ and $\langle\Delta \varepsilon\rangle_{\Omega_{1}}$, compute the tangent modulus $\mathbb{C}_{1} \equiv \mathbb{C}_{1_{(n+1)}}$ 
from the nonlinear constitutive law of the inclusion.

(3) Compute the average strain in the matrix by (2):

$$
\langle\Delta \varepsilon\rangle_{\Omega_{0}}=\frac{\Delta \bar{\varepsilon}-f_{1}\langle\Delta \varepsilon\rangle_{\Omega_{1}}}{1-f_{1}}
$$

(4) Given $\left\langle\varepsilon_{n}\right\rangle_{\Omega_{0}}$ et $\langle\Delta \varepsilon\rangle_{\Omega_{0}}$, compute the tangent modulus $\mathbb{C}_{0} \equiv \mathbb{C}_{0_{(n+1)}}$ from the nonlinear constitutive law of the matrix.

(5) Extract the isotropic part $\mathbb{C}_{0}^{i s o}$ from $\mathbb{C}_{0}$ (see a justification and details in $[4])$.

(6) Compute the Eshelby tensor $\mathbb{E}$ for $\mathbb{C}_{0}^{i s o}$

(7) Compute $\mathbb{C}_{0}$ and $\mathbb{C}_{1}$ at time $t_{n+\alpha}$ :

$$
\left.\left.\mathbb{C}_{i_{(n+\alpha)}}=(1-\alpha) \mathbb{C}_{i_{(n)}}+\alpha \mathbb{C}_{i_{(n+1)}} \quad i=0,1, \quad \alpha \in\right] 0,1\right]
$$

(8) Compute the concentration tensor $\mathbb{B}^{\epsilon}$ by

$$
\mathbb{B}^{\epsilon}=\left\{\mathbb{I}+\mathbb{E}:\left[\mathbb{C}_{0_{(n+\alpha)}^{-1}}: \mathbb{C}_{1_{(n+\alpha)}}-\mathbb{I}\right]\right\}^{-1}
$$

(9) Check compatibility of the average strain in the inclusion by computing the residual:

$$
\mathbf{R}=\mathbb{B}^{\epsilon}:\left[f_{1} \mathbb{B}^{\epsilon}+\left(1+f_{1}\right) \mathbb{I}\right]^{-1}: \Delta \bar{\varepsilon}-\langle\Delta \varepsilon\rangle_{\Omega_{1}} .
$$

(10) $\mathbf{I F}\|\mathbf{R}\| \leq T O L$ THEN END of iterations

(11) ELSE GO TO (1) with the new average strain in the inclusion

$$
\left.\left.\langle\Delta \varepsilon\rangle_{\Omega_{1}}^{k+1}=\langle\Delta \varepsilon\rangle_{\Omega_{1}}^{k}+\xi \mathbf{R}, \quad \xi \in\right] 0,1\right] .
$$

- At convergence, compute the effective tangent modulus $\overline{\mathbb{C}}_{n+\alpha}$ and the macroscopic stress by:

$$
\begin{aligned}
& \overline{\mathbb{C}}_{n+\alpha}=\left[f_{1} \mathbb{C}_{1_{(n+\alpha)}}: \mathbb{B}^{\epsilon}+\left(1-f_{1}\right) \mathbb{C}_{0_{(n+\alpha)}}\right]:\left[f_{1} \mathbb{B}^{\epsilon}+\left(1-f_{1}\right) \mathbb{I}\right]^{-1}, \\
& \Delta \overline{\boldsymbol{\sigma}}=\overline{\mathbb{C}}_{n+\alpha}: \Delta \overline{\boldsymbol{\varepsilon}} \\
& \overline{\boldsymbol{\sigma}}_{n+\alpha}=(1-\alpha) \overline{\boldsymbol{\sigma}}_{n}+\alpha \Delta \overline{\boldsymbol{\sigma}}
\end{aligned}
$$

\section{Proposed methodology to determine the size of the RVE}

\subsection{Description of the procedure}

The procedure to determine the RVE's size is described as follows.

We define a set of volumes $\chi_{V}=\left\{V^{k}\right\}_{k=1}^{K}$, where $K$ is determined by the tolerance criterion defined in (18)-(22), and $V^{1}$ the minimal size of the unit cell. The volume is given by $V=L^{2} \times H, L$ denoting the length of the square domain associated with $\Omega(V)$ and $H$ has a unitary length. The boundary of $\Omega(V)$ is denoted by $\partial \Omega(V)$

For each volume $V^{k}$, we assume that the geometry of the microstructure is modeled by a $\mathbb{R}^{m}$ - valued random variable with probability distribution $P_{\xi}$ for which its support is a subset of $\mathbb{R}^{m}$. The random possible geometrical parameters are represented by the random vector $\boldsymbol{\xi}$. The independent real- 


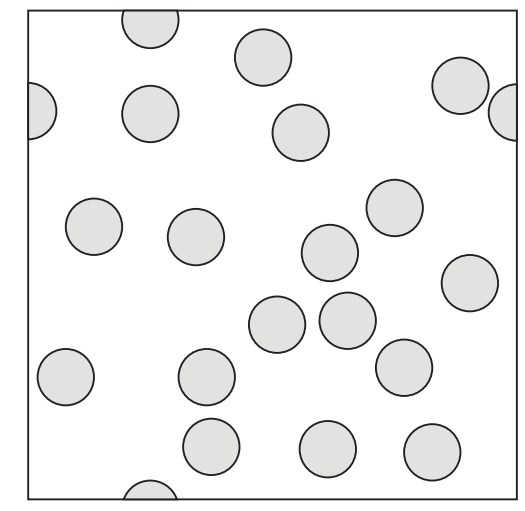

Figure 1. Example of realization of the microstructure.

izations $\chi_{\xi}=\left\{\boldsymbol{\xi}^{r}\right\}_{r=1}^{R}$ of random vector $\boldsymbol{\xi}$ are generated here by a uniform distribution of inclusions centers within $\Omega$, with rejecting trial positions inducing intersection with previously introduced inclusions. An illustration of one realization of the microstructure is provided in Fig. 1. The constitutive law in each phase is nonlinear. Then, $R$ realizations of the microstructure morphology are generated, $R$ being determined by the criterion defined in (23). For each realization $r$, a direct finite element computation is conducted, by prescribing a macroscopic strain load history $\bar{\varepsilon}(t)$ on the RVE through periodic boundary conditions:

$$
\mathbf{u}(t)=\overline{\boldsymbol{\varepsilon}}(t) \mathbf{x}+\tilde{\mathbf{u}}(\mathbf{x}, t) \quad \text { on } \partial \Omega\left(V^{k}\right),
$$

with $\tilde{\mathbf{u}}(\mathbf{x}, t)$ a periodic fluctuation. Defining a time-stepping $\mathcal{T}=\left[t^{1}, t^{2}, \ldots, T\right]$ and solving the nonlinear problem for $t \in[0, T]$, the effective stress response is computed by

$$
\overline{\boldsymbol{\sigma}}\left(V^{k}, \boldsymbol{\xi}^{r}, t\right)=\frac{1}{V^{k}} \int_{\Omega\left(V^{k}\right)} \boldsymbol{\sigma}\left(V^{k}, \boldsymbol{\xi}^{r}, t, \mathbf{x}\right) d \Omega
$$

where $\boldsymbol{\sigma}\left(V^{k}, \boldsymbol{\xi}^{r}, t, \mathbf{x}\right)$ is the local stress in the RVE at time $t$, for a volume $V^{k}$, and for the realization $r$. This solution constitutes the reference solution for the set $\{k, r\}$. Then, choosing an appropriate analytical constitutive law defined by $P$ coefficients $\boldsymbol{\alpha}=\left\{\alpha_{1}, \alpha_{2}, \ldots, \alpha_{P}\right\}$, the incremental procedure described in the previous section is conducted, where the behavior of the inclusions is determined by fixed values of coefficients, but where the behavior of the matrix is associated with the coefficients $\boldsymbol{\alpha}$. The macroscopic stress response can be efficiently evaluated by (14) and is denoted by $\hat{\boldsymbol{\sigma}}\left(V^{k}, \boldsymbol{\xi}^{r}, t\right)$.

Then, the coefficients $\boldsymbol{\alpha}\left(\boldsymbol{\xi}^{r}, V^{k}\right)$ are adjusted so as to minimize the error function:

$$
e=\int_{0}^{T}\left\|\overline{\boldsymbol{\sigma}}\left(V^{k}, \boldsymbol{\xi}^{r}, t\right)-\hat{\boldsymbol{\sigma}}\left(V^{k}, \boldsymbol{\xi}^{r}, t\right)\right\|^{2} d t
$$




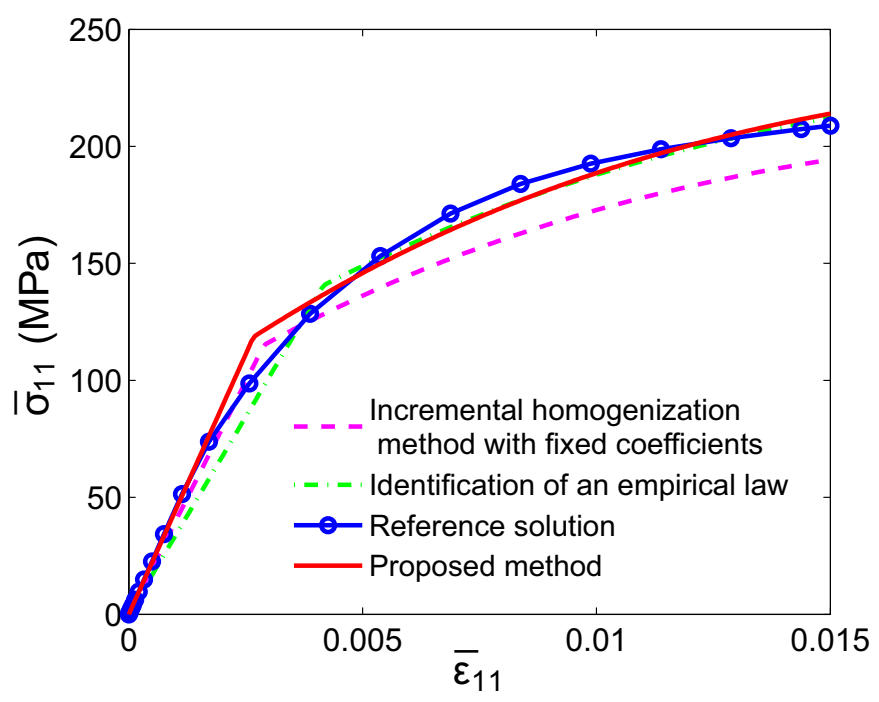

Figure 2. Identification of the numerical parameters $\boldsymbol{\alpha}$ by three approaches for an elastoplastic behavior, and volume fraction $f=0.5$.

with $\|\mathbf{e}\|^{2}=\mathbf{e}: \mathbf{e}$.

For one given volume $V^{k}$, the operation is repeated for $R$ realizations, until a convergence criterion, defined in the next section, is reached. The associated converged parameters related to the analytical constitutive law of the matrix in the incremental homogenization scheme are denoted by $\boldsymbol{\alpha}\left(V^{k}\right)$.

Then, the volume is increased to $V^{k+1}$ and the former steps are repeated. Finally, the convergence is checked with respect to the volume $k$, as defined in the next section. The volume associated to convergence with respect to both sizes $K$ and realizations $R$ gives an estimate for the RVE size, denoted by $V=V^{K, R}$.

\subsection{Convergence criteria}

\subsubsection{Convergence of empirical parameters for a given volume}

For a given volume $V^{k}$, the convergence of the $P$ parameters $\alpha_{j}\left(V^{k}\right), j=$ $1, \ldots, P$ is determined by a simple criterion:

$$
\epsilon_{r e l}=\frac{2 D_{\alpha}^{j}}{M_{\alpha}^{j} \sqrt{R}}
$$

where $R$ denotes the number of independent realizations for the volume $V^{k}$ fixed, for $r$. Let $\chi_{\alpha}^{j}$ be the vector collecting the $R$ independent realizations of 
the parameter $\alpha_{j}$ :

$$
\chi_{\alpha}^{j}=\left\{\alpha_{j}^{1}\left(V^{k}\right), \alpha_{j}^{2}\left(V^{k}\right), \ldots, \alpha_{j}^{R}\left(V^{k}\right)\right\} .
$$

In (19), $M_{\alpha}^{j}$ and $D_{\alpha}^{j}$ denote mean and standard deviations of $\alpha_{j}$ :

$$
\begin{aligned}
& M_{\alpha}^{j}\left[\chi_{\alpha}^{j}\right]=\frac{1}{R} \sum_{r=1}^{R} \alpha_{j}^{r}\left(V^{k}\right) \equiv \mu^{j}, \\
& D_{\alpha}^{j}\left[\chi_{\alpha}^{j}\right]=\sqrt{M_{\alpha}^{j}\left[\left(\chi_{\alpha}^{j}-\mu^{j}\right)^{2}\right]} .
\end{aligned}
$$

The convergence criterion is chosen such that

$$
\epsilon_{\text {rel }} \leq \epsilon_{\text {rel }}^{*}
$$

where $\epsilon_{r e l}^{*}$ is a given tolerance error. In the present work, we choose $\epsilon_{r e l}^{*}=0.05$.

\subsubsection{Convergence of empirical parameters with respect to the volume}

Different approaches have been proposed previously to determine convergence criteria related to the convergence with respect to the volume of the RVE, and associated estimates for the RVE volume (see Kanit et al. [15], Gitman et al. [9], or Pelissou et al. [20]). In [15], the estimation of the variance with respect to the volume size was modeled by a power law, requiring the identification of a metric associated with the covariance. In the present work, as the material is fully nonlinear, the convergence of $\boldsymbol{\alpha}(V)$ is simply estimated by a numerical convergence analysis with respect to $V$ as follows:

$$
\frac{\left\|\boldsymbol{\alpha}\left(V^{k+1}\right)-\boldsymbol{\alpha}\left(V^{k}\right)\right\|}{\left\|\boldsymbol{\alpha}\left(V^{k+1}\right)\right\|}<\delta
$$

where $\delta$ is a tolerance parameter.

Remark a more straightforward approach could have been alternatively proposed, consisting in directly identifying the parameters of a chosen empirical law for the homogenized composite, without employing the incremental homogenization procedure to obtain $\hat{\boldsymbol{\sigma}}\left(V^{k}, \boldsymbol{\xi}^{r}, t\right)$. However, we have found two drawbacks for this option: (a) first, without any knowledge about the effective constitutive law, the range of parameters used for the identification can be very large, leading to heavy computations associated to minimizing (17); (b) a classical constitutive law can lead to inaccurate results with respect to the reference solution, as illustrated below in Fig. 2. Another option would be to analyze the macroscopic stress response of the composite by applying directly the incremental homogenization procedure, and by 
using a constitutive law with fixed values for the matrix. As shown below, this can also lead to inaccurate results with respect to the reference solution. For illustration, we compare in Fig. 2 the effective stress response obtained by a direct computation by FEM (reference solution) for the nonlinear composite described in section 4 with the following estimates: i) the stress response obtained by directly applying the incremental procedure; ii) the stress response obtained by identifying an empirical constitutive law without the incremental homogenization scheme and iii), the stress response obtained by the proposed corrected incremental homogenization scheme. We can note that the last solution is the closest to the reference solution. The case corresponds to the case of elastic inclusions in an elastoplastic matrix, for a volume fraction $f=0.5$ and material parameters provided in Table 1 , except that the Young moduli of inclusions and matrix are XX and YY, respectively. Furthermore, this last strategy is also the most efficient, because in that case the range for the parameters to be identified can be set much narrower.

\section{Numerical examples}

In the following two examples, a 2D RVE is considered, containing randomly distributed cylindrical fibers, as depicted in figure 1. The fibers cannot interpenetrate and the inclusions are supposed to be elastic, while the matrix has a nonlinear behavior. We study two cases: an elastoplastic matrix and a elasto-viscoplastic matrix. For each case, two volume fractions are studied: $f=0.3$ and $f=0.5$. For a given number of fibers and a given volume fraction, we define the size of the RVE as the length of one edge of the square domain containing the fibers, $L=\sqrt{(\pi N) / f} D / 2, D$ being the diameters of the inclusions.

\subsection{Composite with elastoplastic matrix}

In this first example, the matrix is assumed to have an elastoplastic behavior, described by $J_{2}$-flow theory with nonlinear isotropic hardening. In that context, the constitutive law is given by:

$$
\boldsymbol{\sigma}=\mathbb{C}:\left(\varepsilon-\varepsilon^{p}\right)
$$

where $\varepsilon^{p}$ is the plastic strain and where $\mathbb{C}$ the elastic tensor, assumed to be isotropic, i.e. $\mathbb{C}=\kappa \mathbf{1} \otimes \mathbf{1}+\mu\left(\mathbb{I}-\frac{1}{3} \mathbf{1} \otimes \mathbf{1}\right)$, with $\kappa$ the bulk modulus expressed by $\kappa=E /(3(1-2 \nu))$ and $\mu$ the shear coefficient expressed by $\mu=E / 2(1+\nu)$. 


\begin{tabular}{lll}
\hline & Matrix & Inclusion \\
\hline Young modulus $G(\mathrm{MPa})$ & 15000 & 300000 \\
Poisson coefficient $\nu$ & 0.2 & 0.45 \\
Yield Stress $\sigma_{Y}(\mathrm{MPa})$ & 80 & \\
Hardening modulus $\sigma_{\infty}(\mathrm{MPa})$ & 60 & \\
Hardening exponent $m$ & 75 & \\
Linear hardening modulus $k(\mathrm{MPa})$ & 20 &
\end{tabular}

Table 1

Material parameters for the matrix and the inclusions for the composite with elastplastic model.

In the context of the $J_{2}$ theory, the response is assumed to be linear if

$$
f(\boldsymbol{\sigma}, p)=J_{2}(\boldsymbol{\sigma})-\sigma_{Y}-R(p) \leq 0
$$

where $J_{2}(\boldsymbol{\sigma})=\left(\frac{3}{2} \mathbf{s}: \mathbf{s}\right), \mathbf{s}=\boldsymbol{\sigma}-\frac{1}{3} \operatorname{Tr}(\boldsymbol{\sigma}), \operatorname{Tr}($.$) being the trace operator, \sigma_{Y}$ is the yield stress, $R(p)$ is the hardening stress and $p$ the cumulated equivalent plastic strain express by:

$$
p(t)=\int_{0}^{t} \dot{p}(\tau) d \tau
$$

with $\dot{p}=\left(\frac{2}{3} \dot{\varepsilon}^{p}: \dot{\varepsilon}^{p}\right)^{1 / 2}$.

The evolution of the plastic strain $\varepsilon^{p}$ is given by the normality rule:

$$
\dot{\boldsymbol{\varepsilon}}^{p}=\dot{p} \frac{\partial f}{\partial \boldsymbol{\sigma}}
$$

In this work, the hardening strain is assumed to be in the form:

$$
R(p)=k p+\sigma_{\infty}\left(1-e^{-m \alpha}\right)
$$

where $k$ is the linear hardening modulus and $\sigma_{\infty}$ is the hardening modulus, and $m$ is hardening exponent. The numerical parameters of the model are indicated in Table 1.

As described in the previous sections, Finite Element computations are performed by prescribing a tensile loading $\bar{\varepsilon}=\bar{\varepsilon}_{11}(t) \mathbf{e}_{1} \otimes \mathbf{e}_{1}$ on the RVE, in order to provide a reference solution. Illustrations of some von Mises stress fields for cases involving 4 and 144 inclusions, with volume fractions $f=0.3$ and $f=0.5$ are provided in Figs. 3 and 4 . We have observed highly localized shear bands for small values of $L / D$. However, for much larger values of $L / D$, these bands exist but are stopped by surrounding inclusions and remain limited. 


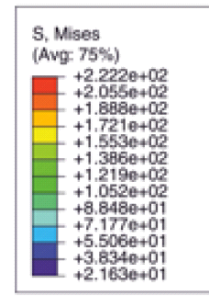

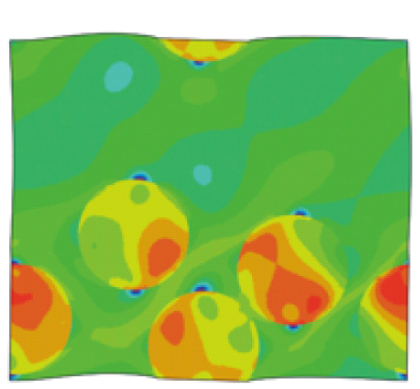

(a)

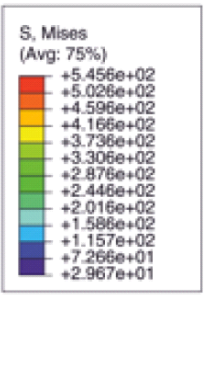

(b)

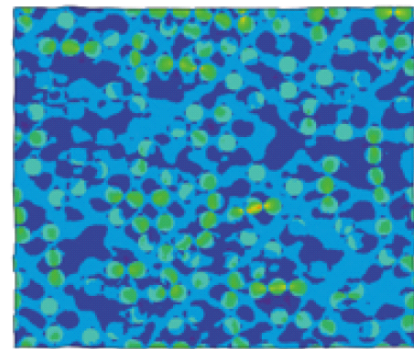

Figure 3. Elastoplastic composite: von Mises stress field in the case of volume fraction $f=0.3$ : (a) 4 inclusions, corresponding to $L / D=3.23$; (b) 144 inclusions, corresponding to $L / D=19.41$

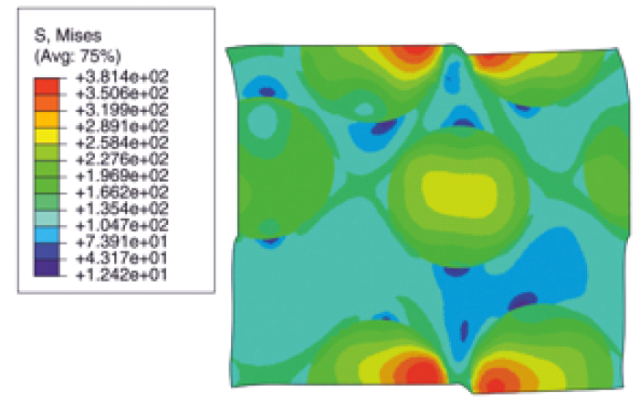

(a)

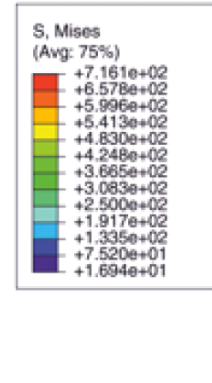

(b)

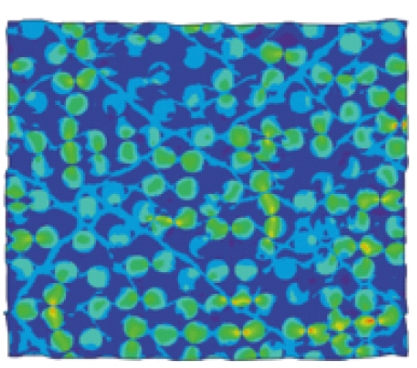

Figure 4. Elastoplastic composite: von Mises stress field in the case of volume fraction $f=0.5$ : (a) 4 inclusions, , corresponding to $L / D=2.5066$; (b) 144 inclusions, corresponding to $L / D=15.03$

This induces a lack of ergodicity of the process, which requires high sizes of RVE (ratio $L / D$ ) to obtain converged values of parameters, as shown in the following. This phenomenon has also been shown in [8].

The procedure described in section 3 is carried out for the two cases, $f=0.3$ and $f=0.5$. The statistical convergence of the parameters $\sigma_{Y}, \sigma_{\infty}, m$ and $k$ is depicted in Figs. 5-6. The respective errors $\epsilon_{r e l}^{*}$ have been chosen respectively as $\epsilon_{r e l}^{*}=0.02, \epsilon_{r e l}^{*}=0.02, \epsilon_{r e l}^{*}=0.03$ and $\epsilon_{r e l}^{*}=0.04$.

In figures 7-10, the convergence of the parameters with respect to the RVE size $L / D$ is depicted. For each value of $L / D$, we have reported the converged value with respect to the number of realization $R$.

In the case of the volume fraction $f=0.3$, we can observe a convergence of the different parameters for RVE size of roughly $L / D=17-18$. In the case $f=0.5$, the RVE size is roughly $L / D=14-15$. 


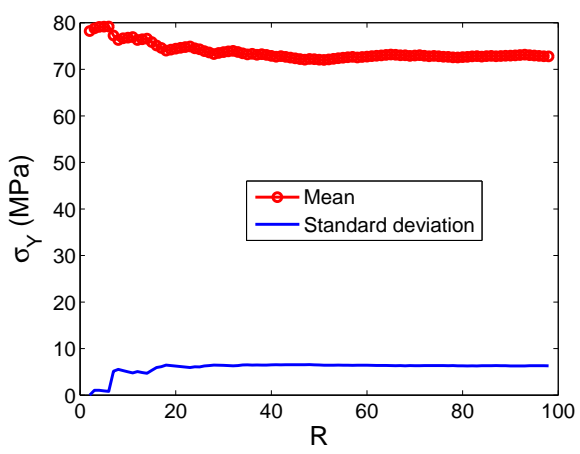

(a)

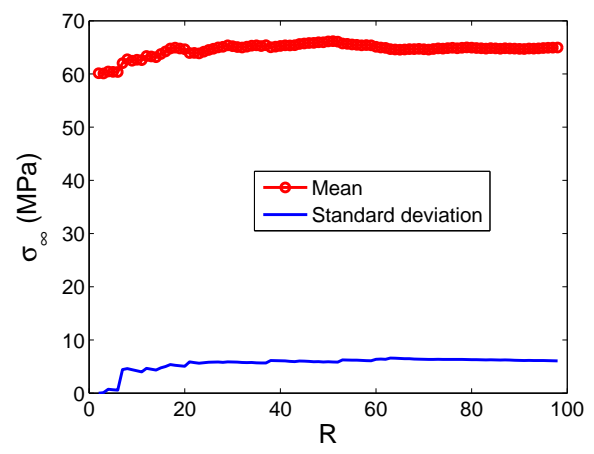

(b)

Figure 5. Elastoplastic composite, $f=0.3$ : statistical convergence of parameters: (a) $\sigma_{Y}$ and (b) $\sigma_{\infty}$ for a fixed size of RVE $L / D=3.23$.

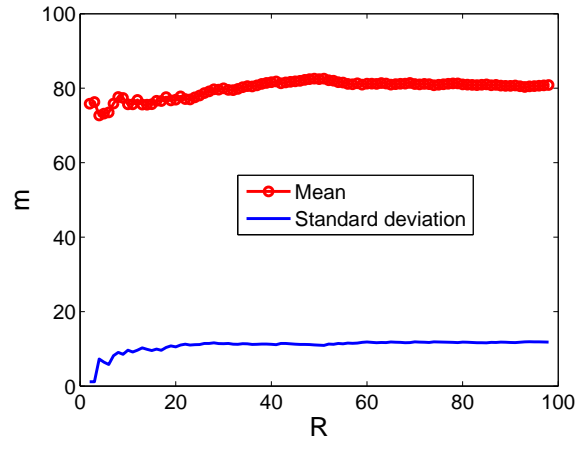

(a)

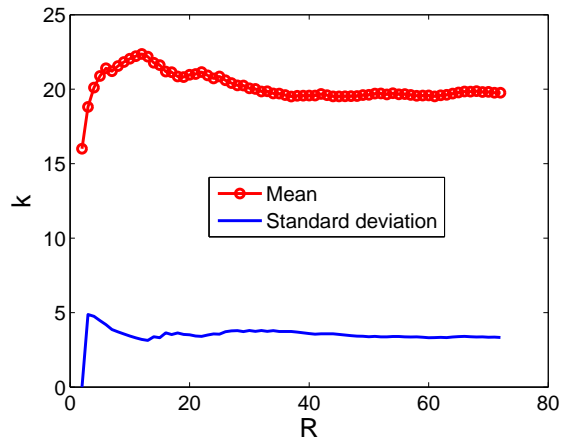

(b)

Figure 6. Elastoplastic composite, $f=0.3$ : statistical convergence of parameters: (a) $m$ and (b) $k$ for a fixed size of $\operatorname{RVE} L / D=3.23$.

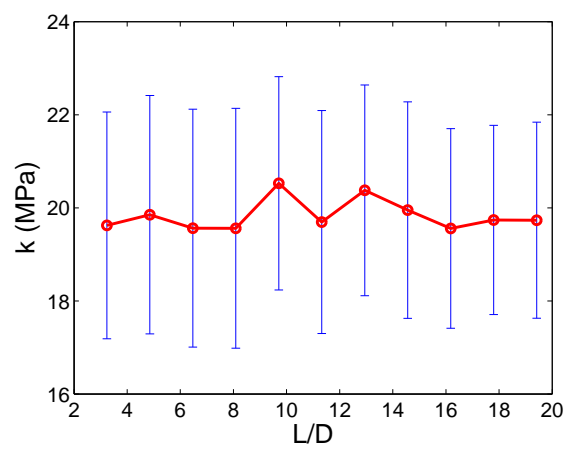

(a)

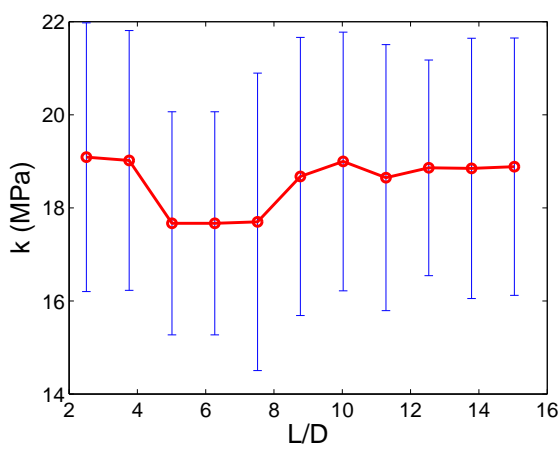

(b)

Figure 7. Elastoplastic composite: convergence of the parameter $k$ with respect to the volume of the RVE, or equivalently with respect to $L / D$; (a) volume fraction $f=0.3$; (b) volume fraction $f=0.5$. 


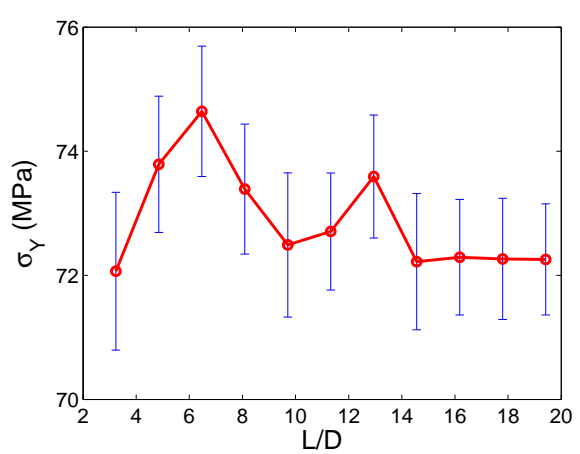

(a)

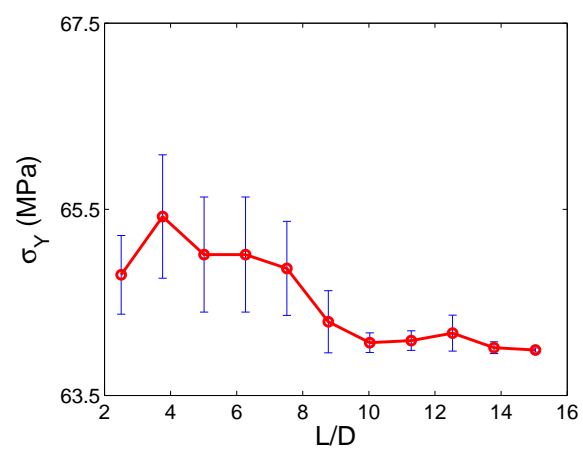

(b)

Figure 8. Elastoplastic composite: convergence of the parameter $\sigma_{Y}$ with respect to the volume of the RVE, or equivalently with respect to $L / D$; (a) volume fraction $f=0.3 ;$ (b) volume fraction $f=0.5$.

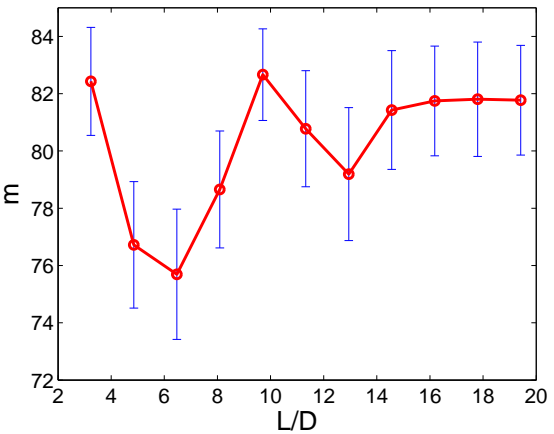

(a)

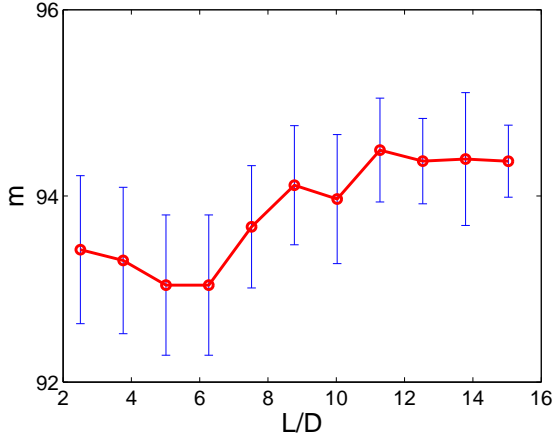

(b)

Figure 9. Elastoplastic composite: convergence of the parameter $m$ with respect to the volume of the RVE, or equivalently with respect to $L / D$; (a) volume fraction $f=0.3 ;$ (b) volume fraction $f=0.5$.

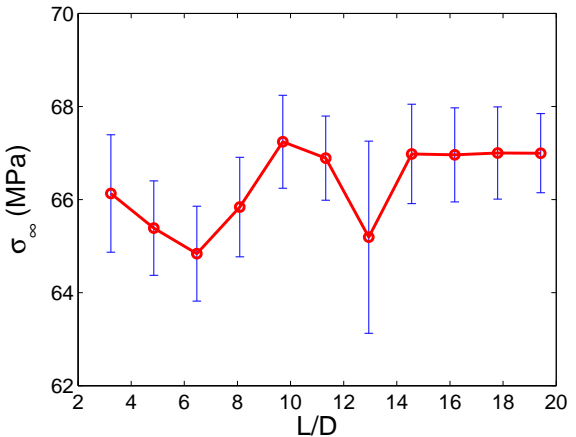

(a)

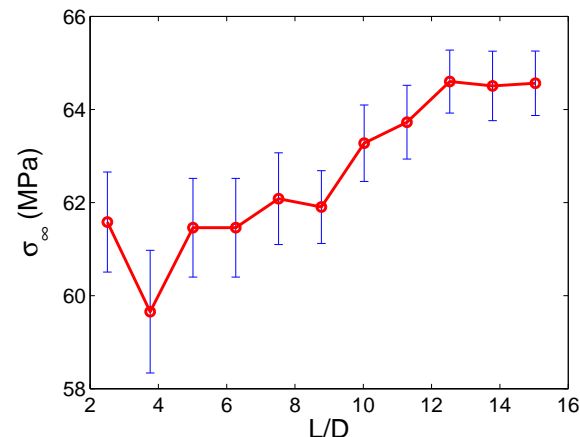

(b)

Figure 10. Elastoplastic composite: convergence of the parameter $\sigma_{\infty}$ with respect to the volume of the RVE, or equivalently with respect to $L / D$; (a) volume fraction $f=0.3 ;$ (b) volume fraction $f=0.5$. 


\section{2 composite with Elasto-viscoplastic matrix}

In this second example, the matrix is assumed to have an elasto-viscoplastic behavior. The total strain $\varepsilon$ is assumed to be the sum of an elastic part and a viscoplastic part $\varepsilon^{v p}$ as:

$$
\varepsilon=\varepsilon^{e}+\varepsilon^{v p} .
$$

The viscous effects are only considered in the plasticity domain. The evolution of the viscoplastic strain is given by the flow rule:

$$
\dot{\varepsilon}^{v p}=\dot{p} \frac{\partial f}{\partial \boldsymbol{\sigma}}
$$

and the stress satisfies (25). In this work, a current yield stress Norton law [19] has been chosen to describe the evolution of $\dot{p}=\frac{d p}{d t}$ :

$$
\dot{p}=\frac{\sigma_{Y}}{\eta}\left(\frac{f}{\sigma_{Y}+\sigma(p)}\right)^{s}
$$

where $\eta$ is the viscoplastic Norton coefficient. This parameter indicates the viscoplastic sensitivity of the material to the strain rate, $s$ is the viscoplastic exponent and $f$ is the viscoplastic part of the stress, defined as :

$$
f=J_{2}(\sigma)-\sigma_{Y}-R(p) .
$$

The numerical values for these parameters are indicated in Table 2.

Here again, the procedure described in section 3 is carried out. Illustrations of von Mises stress fields obtained during the computation of the reference solution are depicted in figures 11 and 12 , corresponding to $f=0.3, f=0.5$, respectively, for cases involving $N=4$ and $N=144$ inclusions.

In this case of an elasto-viscoplastic composite, we also observe shear bands, which propagate outside of the cell for small sizes $L / D$ and are blocked for larger sizes. However, these bands are much less localized in the viscoplastic case and induce a faster convergence with respect to the size $L / D$, and thus leading to smaller RVE sizes. 


\begin{tabular}{lll}
\hline & Matrix & Inclusion \\
\hline Young modulus $G(\mathrm{MPa})$ & 15000 & 300000 \\
Poisson coefficient $\vartheta$ & 0.2 & 0.45 \\
Yield Stress $\sigma_{Y}(\mathrm{MPa})$ & 80 & \\
Hardening modulus $\sigma_{\infty}(\mathrm{MPa})$ & 60 & \\
Hardening exponent $m$ & 75 & \\
Linear hardening modulus $k(\mathrm{MPa})$ & 20 & \\
Viscoplastic coefficient $\eta$ & 2 & \\
Viscoplastic exponent $s$ & 100 & \\
\hline
\end{tabular}

Table 2

Material parameters for the matrix and the inclusions for the composite with elastoviscoplastic model

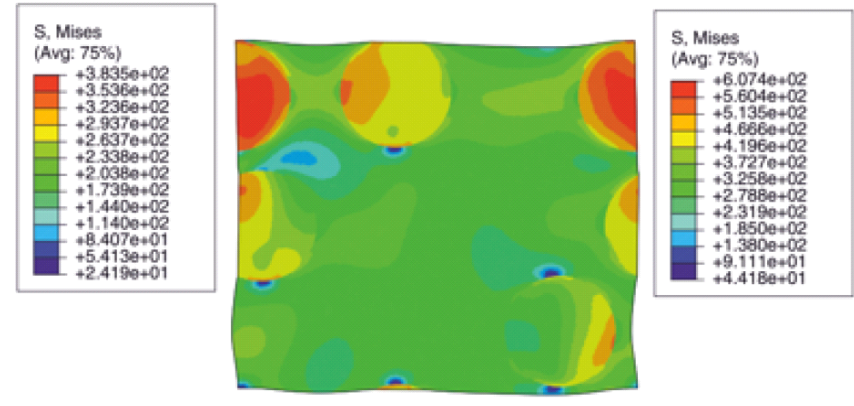

(a)

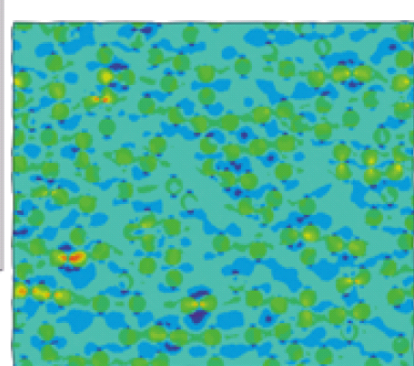

(b)

Figure 11. Elasto-viscoplastic composite: von Mises stress field in the case of volume fraction $f=0.3$ : (a) 4 inclusions, corresponding to $L / D=3.23$; (b) 144 inclusions, corresponding to $L / D=19.41$

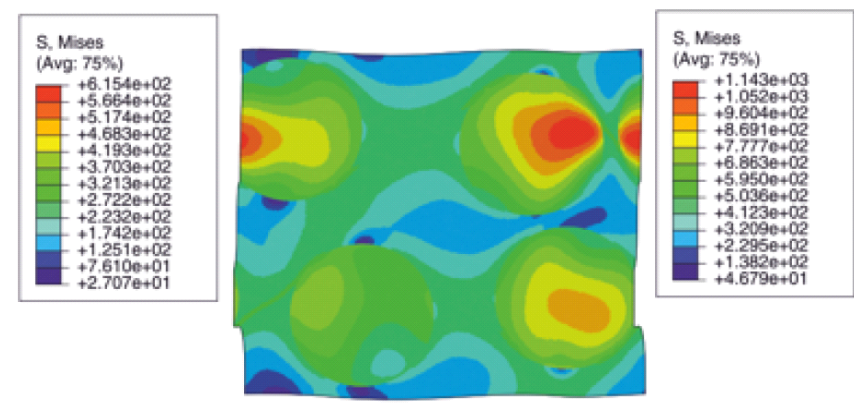

(a)

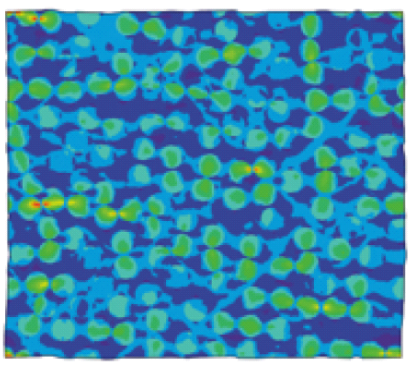

(b)

Figure 12. Elasto-viscoplastic composite: von Mises stress field in the case of volume fraction $f=0.5$ : (a) 4 inclusions, , corresponding to $L / D=2.5066$; (b) 144 inclusions, corresponding to $L / D=15.03$ 


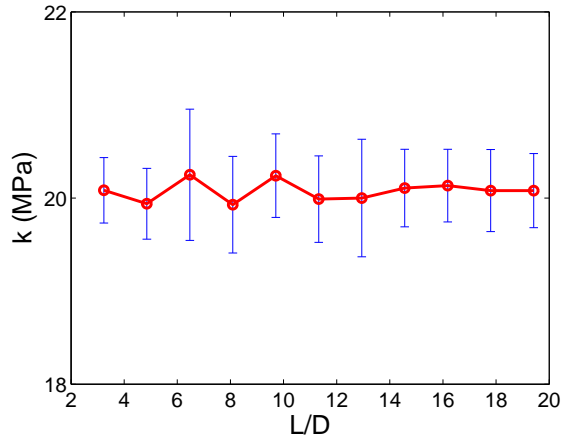

(a)

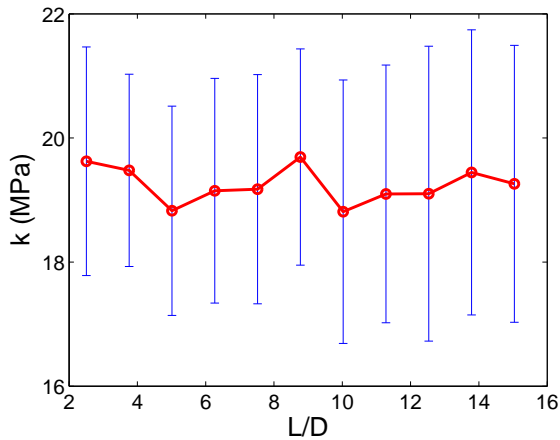

(b)

Figure 13. Elasto-viscoplastic composite: convergence of the parameter $k$ with respect to the volume of the RVE, or equivalently with respect to $L / D$; (a) volume fraction $f=0.3$; (b) volume fraction $f=0.5$.

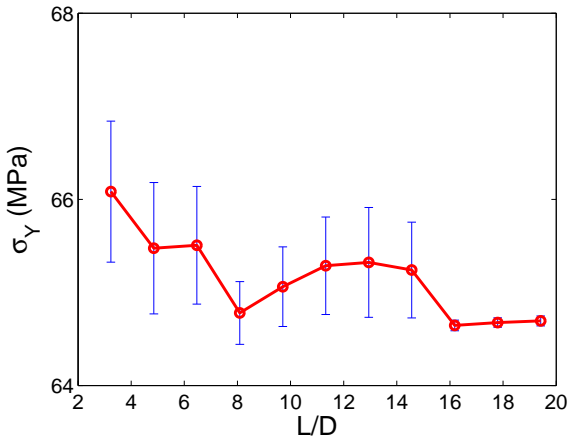

(a)

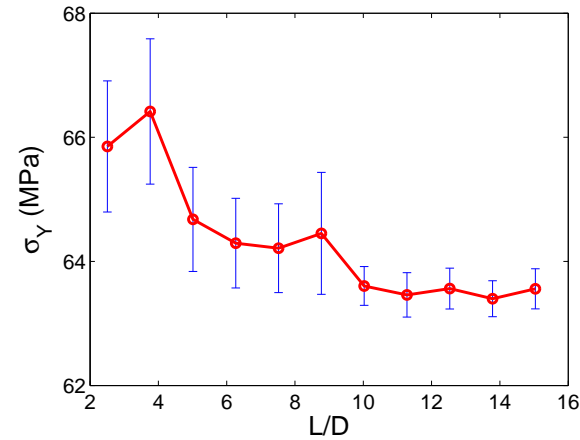

(b)

Figure 14. Elasto-viscoplastic composite: convergence of the parameter $\sigma_{Y}$ with respect to the volume of the RVE, or equivalently with respect to $L / D$; (a) volume fraction $f=0.3$; (b) volume fraction $f=0.5$.

In figures $13-18$, the convergence of the parameters with respect to $L / D$ is depicted, as in the previous example.

We can note that the convergence of the hardening mudulus $k$ and of the viscoplastic coefficient $\eta$ is quite fast with respect to the size $L / D$, while the other coefficients converge much slower. Eventually, the RVE size $L / D$ for which a convergence is observed with respect to all parameters is in this case about $L / D=14-15$. The faster convergence in the case of composites with elasto-viscoplastic matrix can be explained by less localization in the matrix as compared to the elastoplastic case. 


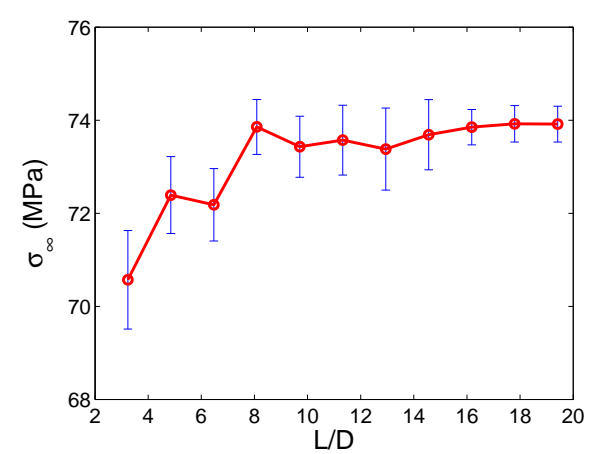

(a)

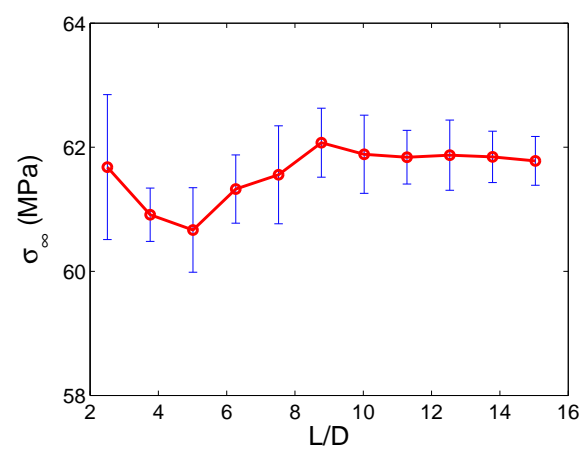

(b)

Figure 15. Elasto-viscoplastic composite: convergence of the parameter $\sigma_{\infty}$ with respect to the volume of the RVE, or equivalently with respect to $L / D$; (a) volume fraction $f=0.3$; (b) volume fraction $f=0.5$.

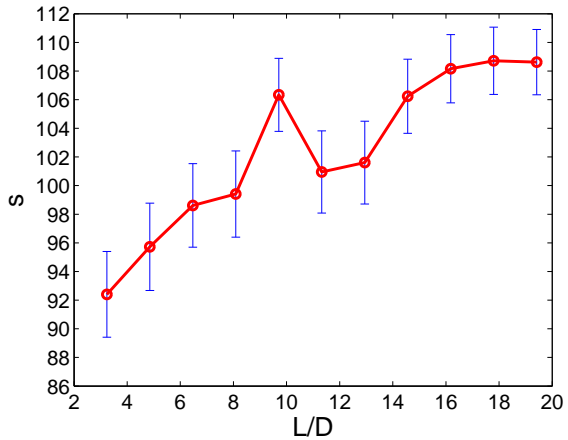

(a)

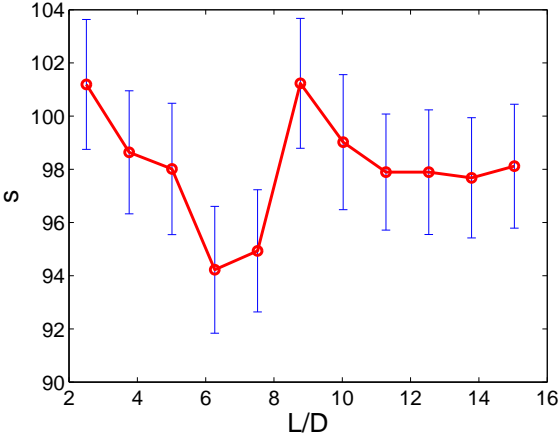

(b)

Figure 16. Elasto-viscoplastic composite: convergence of the parameter $s$ with respect to the volume of the RVE, or equivalently with respect to $L / D$; (a) volume fraction $f=0.3$; (b) volume fraction $f=0.5$.

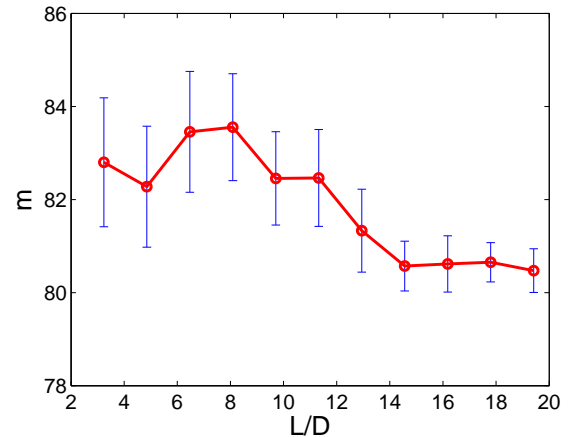

(a)

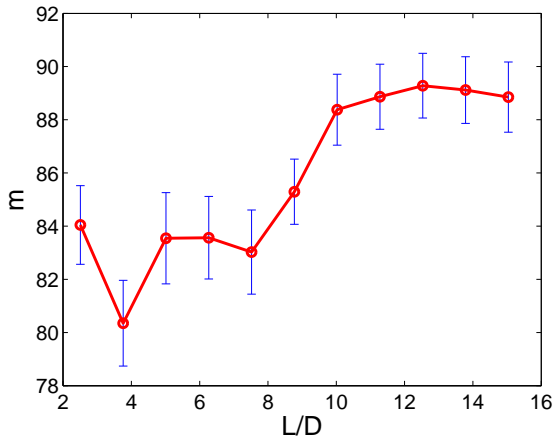

(b)

Figure 17. Elasto-viscoplastic composite: convergence of the parameter $m$ with respect to the volume of the RVE, or equivalently with respect to $L / D$; (a) volume fraction $f=0.3$; (b) volume fraction $f=0.5$. 


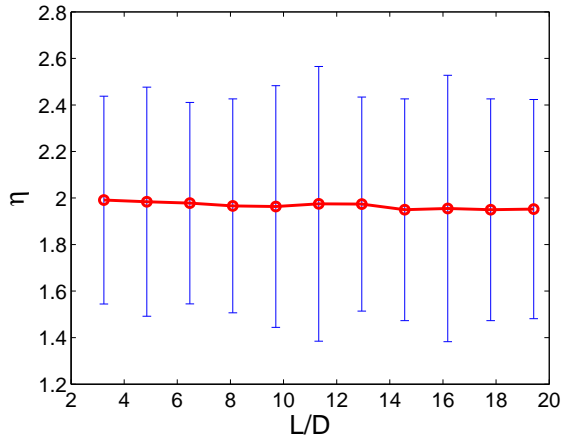

(a)

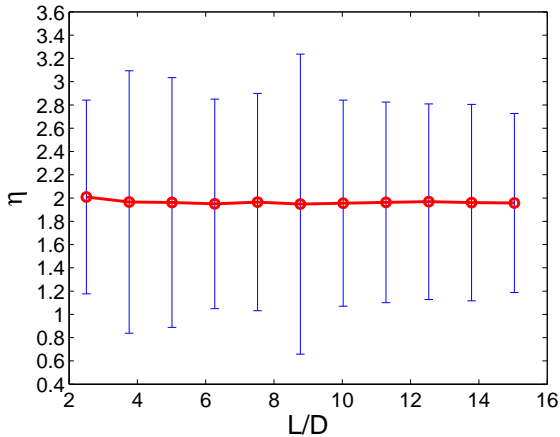

(b)

Figure 18. Elasto-viscoplastic composite: convergence of the parameter $\eta$ with respect to the volume of the RVE, or equivalently with respect to $L / D$; (a) volume fraction $f=0.3$; (b) volume fraction $f=0.5$.

\section{Conclusion}

A new procedure to determine the size of RVE for nonlinear random materials has been proposed. The technique is based on analyzing the convergence of parameters used in an incremental homogenization framework [4] and identified by means of FEM nonlinear finite element computations, with respect to both statistical realization of the random microstructure and to the size of the unit cell. Two cases were studied, involving fiber-composites with hard elastic cylindrical fibers and elastoplastic and elasto-viscoplastic matrix, for different volume fractions. We have found a convergence of the parameters leading to the existence of an RVE for these cases, with a size estimated as 17-18 and 13-15 times the diameter of the inclusions for the elastoplastic and viscoplastic cases, respectively. The faster convergence in the case of composites with elasto-viscoplastic matrix can be explained by less localization in the matrix as compared to the elastoplastic case. Once the RVE size is identified, the related parameter used in the incremental homogenization framework provide a very accurate homogenization model, useable in efficient structure calculations.

\section{Aknowledgements}

The financial support this work enjoys from Ile-de France Region, BpiFrance, DGE and ILMAB Team is gratefully aknowledged. 


\section{References}

[1] W.L. Azoti, A. Tchalla, Y. Koutsawa, A. Makradi, G. Rauchs, S. Bellouetar, and H. Zahrouni. Mean-field constitutive modeling of elasto-plastic composites using two (2) incremental formulations. Compos. Struct., 105:256-262, 2013.

[2] V. Bulsara, R. Talreja, and J. Qu. Damage initiation under transverse loading of unidirectional composites with arbitrarily distributed fibers. Compos. Sci. Technol., 59:673-682, 1999.

[3] J. Cugnoni and M. Galli. Representative volume element size of elastoplastic and elastoviscoplastic particle-reinforced composites with random microstructure. Comput. Model. Eng. Sci., 66(2):165-185, 2010.

[4] I. Doghri and A. Ouaar. Homogenization of two-phase elasto-plastic composite materials and structures, study of tangent operators, cyclic plasticity and numerical algorithms. Int. J. Sol. Struct., 40:1681-1712, 2003.

[5] X. Du and M. Ostoja-Starzewski. On the size of representative volume element for darcy law in random media. Proc. R. Soc. A, 462:2949-2963, 2006.

[6] G. Dvorak. Micromechanics of Composites Materials. Springer, New-York, 2013.

[7] A. Elvin and S.S. Sunder. Microcracking due to grain boundary sliding in polycrystalline ice under uniaxial compression. Acta Mater., 44(1):43-56, 1996.

[8] F. Fritzen, S. Forest, T. Böhlke, D. Kondo, and T. Kanit. Computational homogenization of elasto-plastic porous metals. Int. J. Plast., 29(0):102 - 119, 2012 .

[9] I.M. Gitman, H. Askes, and L.J. Sluys. Representative volume: Existence and size determination. Eng. Frac. Mech., 74(16):2518 - 2534, 2007.

[10] C. Gonzalez and J. LLorca. Mechanical behavior of unidirectional fiberreinforced polymers under transverse compression: Microscopic mechanisms and modeling. Compos. Sci. Technol., 67(13):2795 - 2806, 2007.

[11] Q. Grimal, K. Raum, A. Gerisch, and P. Laugier. A determination of the minimum sizes of representative volume elements for the prediction of cortical bone elastic properties. Biomech. Model. Mechanobiol., 10:925-937, 2011.

[12] A.A. Gusev. Representative volume element size for elastic composites: a numerical study. J. Mech. Phys. Solids, 45:1449 -1459, 1997.

[13] C. Heinrich, M. Aldridge, A.S. Wineman, J. Kieffer, A.M. Waas, and K. Shahwan. The influence of the representative volume element (rve) size on the homogenized response of cured fiber composites. Modelling Simul. Mater. Sci. Eng., 20:075007, 2012.

[14] R. Hill. A self-consistent mechanics of composites materials. J. Mech. Phys. Solids, 13:213-222, 1965. 
[15] T. Kanit, S. Forest, I. Galliet, V. Mounoury, and D. Jeulin. Determination of the size of the representative volume element for random composites: statistical and numerical approach. Int. J. Solids Struct., 40(13-14):3647 - 3679, 2003.

[16] Z. F. Khisaeva and M. Ostoja-Starzewski. On the size of rve in finite elasticity of random composites. J. elasticity, 37, 2006.

[17] I. Monetto and W.J. Drugan. A micromechanics-based non local constitutive equation and minimum rve size estimates for random elastic composites containing aligned spheroidal heterogeneities. J. Mech. Phys. Solids, 57:1578$1595,2009$.

[18] M. Ostoja-Starzewski. Material spatial randomness: from statistical to representative volume element. Probab. Eng. Mech., 21:112-132, 2006.

[19] N.S. Ottosen and M. Ristinmaa. Mechanics of Constitutive Modeling. Elsevier Science Ltd, 2005.

[20] C. Pelissou, J. Baccou, Y. Monerie, and F. Perales. Determination of the size of the representative volume element for random quasi-brittle composites. Int. J. Solids Struct., 46(14-15):2842 - 2855, 2009.

[21] V. Pensée and Q.-C. He. Generalized self-consistent estimation of the apparent isotropic elastic moduli and minimum representative volume element size of heterogeneous media. Int. J. Sol. Struct., 44(7):2225-2243, 2007.

[22] J.F. Rakow and A.M. Waas. Size effects in metal foam cores for sandwich structures. AIAA J., 42:7, 2004.

[23] I.S. Ranganathan and M. Ostoja-Starzewski. Scaling function, anisotropy and the size of rve in elastic random polycrystals. J. Mech. Phys. Solids, 56:2773$2791,2008$.

[24] Z.Y. Ren and Q.S. Zheng. A quantitative study of minimum sizes of representative volume elements of cubic polycrystals - numerical experiments. J. Mech. Phys. Solids, 50:881-893, 2002.

[25] S. Swaminathan S and S. Ghosh. Statistically equivalent representative volume elements for unidirectional composite microstructures: Ii. with interfacial debonding. J. Compos. Mater., 49:605-621, 2006.

[26] K. Sab and B. Nedjar. Periodization of random media and representative volume element size for linear composites. C. R. Mech., 333:187-195, 2005.

[27] A. Salahouelhadj and H. Haddadi. Estimation of the size of the rve for isotropic copper polycrystals by using elastic-plastic finite element homogenisation. Computat. Mater. Sci., 48(3):447 - 455, 2010.

[28] M. Salmi, F. Auslender, M. Bornert, and M. Fogli. Various estimates of representative volume element sizes based on a statistical analysis of the apparent behavior of random linear composites. C. R. Meca., 340:230-246, 2012. 
[29] J.C. Simo and T.J.R. Hugues. Computational Inelasticity. Springer-Verlag, 1998.

[30] C. Soize. Tensor-valued random fields for meso-scale stochastic model of anisotropic elastic microstructure and probabilistic analysis of representative volume element size. Probab. Eng. Mech., 23:307-323, 2008.

[31] S. Swaminathan, S. Ghosh, and N.J. Pagano. Statistically equivalent representative volume elements for unidirectional composite microstructures: I. without damage. J. Compos. Mater., 40:583-604, 2006.

[32] P.K. Valavala, G.M. Odegard, and E.C. Aifantis. Influence of representative volume element size on predicted elastic properties of polymer materials. Modelling Simul. Mater. Sci. Eng., 17:045004, 2009.

[33] W.J. Drugan J.R. Willis. A micromechanics-based nonlocal constitutive equation and estimates of representative volume element size for elastic composites. J. Mech. Phys. Solids, 44(4):497-524, 1996.

[34] T.I. Zhodi and P. Wriggers. On the sensitivity of homogenized material responses at infinitesimal and finite strains. Commun. Numer. Methods Eng., 16:657-670, 2000. 